\title{
Antiplatelet therapy following TAVI: time to randomise
}

\author{
R. Delewi • J. J. Piek
}

Published online: 8 January 2014

(C) The Author(s) 2014. This article is published with open access at Springerlink.com

Following the introduction of transcatheter aortic valve implantation (TAVI), it is now possible to treat elderly patients with severe aortic stenosis and a high surgical risk. This advancement in interventional cardiology has led to increased survival, health status and quality of life. However, as with many invasive interventional procedures, TAVI is associated with the risk of cerebral embolisation and consequent ischaemic cerebrovascular events with possible neurological impairment. The risk estimates of post-procedural stroke associated with TAVI vary from 1.5 to $10 \%$ and have been reported both early and late after the procedure. In the PARTNER Cohort B study, the Canadian multicentre TAVI registry and the study by Nuis et al., about $50 \%$ of the stroke events occurred more than $24 \mathrm{~h}$ post-procedure [1]. In addition to clinically overt stroke, asymptomatic silent cerebral infarcts have been frequently observed on MRI, with a prevalence ranging from 62 to $93 \%$ [2]. Although asymptomatic, accumulating evidence implicates that these silent cerebral infarcts are related to risk of future stroke, cognitive decline, dementia, and depression. Whether stroke occurs due to incomplete endothelialisation of the valve or by new onset of silent atrial fibrillation is unknown. Early post-procedural stroke $(<24 \mathrm{~h})$ is more likely the result of embolisation during valve implantation.

Given this high incidence of cerebral events, adequate anticoagulation is probably required both during the procedure and in the following months. However, the exact regimen of anticoagulation involves a clinical dilemma with the therapeutic potential of reducing ischaemic complications at the expense of increasing the bleeding risk. The overall incidence of bleeding following TAVI has been reported in $41 \%$ of the

R. Delewi $\cdot$ J. J. Piek $(\bowtie)$

Department of Cardiology, Academic Medical Center, University of Amsterdam, Meibergdreef 9, 1105 AZ Amsterdam, the Netherlands e-mail: j.j.piek@amc.uva.nl patients in the meta-analysis conducted by Généreux et al., of which $16 \%$ were life-threatening [3]. It is important to bear in mind that life-threatening bleeding is associated with a six- to nine-fold increase in 30-day mortality after procedure and acts as an independent predictor of 1-year mortality [4]. Therefore, for clinical decision-making on antithrombotic treatment, it is important to find a balance between prevention of ischaemic complications and avoidance of bleeding risk.

The recently published study by Nijenhuis et al. addresses this clinical dilemma regarding decision-making on anticoagulation treatment of elderly patients treated with TAVI [5]. As pointed out by the authors, the current European and American guidelines merely provide recommendations based on retrospective registries. The European Society of Cardiology guidelines recommend a combination of low aspirin and a thienopyridine early after TAVI, followed by aspirin or a thienopyridine alone. In those cases where there is an indication for treatment with vitamin $\mathrm{K}$ antagonists, such as in atrial fibrillation, a combined treatment of vitamin $\mathrm{K}$ antagonist and aspirin or a thienopyridine is in general installed. However, the guidelines do not provide any recommendations on the duration of dual antiplatelet therapy (DAPT).

As observed in the survey undertaken in the Netherlands, a wide heterogeneity in the duration of dual antiplatelet therapy (DAPT) and loading dose of clopidogrel was noted. In twothirds of the centres $(n=9)$ DAPT was prescribed for 3 months. In one centre, where only transapical TAVI is performed, no concomitant clopidogrel was prescribed at all. The duration of concomitant clopidogrel was dependent on the type of prosthesis in only two centres according to the recommendations of the respective manufactures, 6 months versus 3 months for supra-annular Core Valve ${ }^{\mathrm{TM}}$ and intra-annular prostheses (e.g. SAPIEN $^{\mathrm{TM}}$, JenaValve), respectively. Whether the decision on the duration of concomitant clopidogrel and its loading dose indeed should be dependent on the valve type SAPIEN $^{\mathrm{TM}}$ or Medtronic Core Valve ${ }^{\mathrm{TM}}$ ) or procedural approach 
(transfemoral or apical) has not been described in the literature. Moreover, clinical decision-making becomes more complex in circumstances where there is a high bleeding risk, PCI ( $<1$ year) prior to TAVI, triple therapy pre-TAVI and newonset atrial fibrillation. This is nicely illustrated in the survey of the participating centres showing different approaches to these scenarios.

In conclusion, the appropriate regimen of antiplatelet and anticoagulant therapy following TAVI is undefined and warrants the conduction of randomised controlled trials for clinical decision-making in this steadily growing patient population.

Open Access This article is distributed under the terms of the Creative Commons Attribution License which permits any use, distribution, and reproduction in any medium, provided the original author(s) and the source are credited.

\section{References}

1. Nuis RJ, van Mieghem NM, Schultz CJ, et al. Frequency and causes of stroke during or after transcatheter aortic valve implantation. Am J Cardiol. 2012;109:1637-43.

2. Hassell ME, Nijveldt R, Roos YB, et al. Silent cerebral infarcts associated with cardiac disease and procedures. Nat Rev Cardiol. 2013;10:696-706.

3. Genereux P, Head SJ, van Mieghem NM, et al. Clinical outcomes after transcatheter aortic valve replacement using valve academic research consortium definitions: a weighted meta-analysis of 3,519 patients from 16 studies. J Am Coll Cardiol. 2012;59:2317-26.

4. Borz B, Durand E, Godin M, et al. Incidence, predictors and impact of bleeding after transcatheter aortic valve implantation using the balloon-expandable Edwards prosthesis. Heart. 2013;99:860-5.

5. Nijenhuis VJ, Stella PR, Baan J, et al. Antithrombotic therapy in patients undergoing TAVI: an overview of Dutch hospitals. Neth Heart J 2013. doi:10.1007/s12471-013-0496-6. 